\title{
Posterior Capsular Rent: Risk Factors, Diagnosis and Management
}

\author{
Nimmi Rani ${ }^{*}$, Prashant Gupta ${ }^{2}$ \\ ${ }^{1}$ Aravind Eye Hospital, Madurai, India \\ ${ }^{2}$ Cataract Services at Aravind Eye Hospital, Madurai, India \\ Email: dr.nimmirani@ymail.com, drprashgupta05@gmail.com
}

Received 11 March 2014; revised 10 April 2014; accepted 17 April 2014

Copyright (C 2014 by authors and Scientific Research Publishing Inc.

This work is licensed under the Creative Commons Attribution International License (CC BY).

http://creativecommons.org/licenses/by/4.0/

c) (i) Open Access

\begin{abstract}
In spite of a steady improvement in the safety of cataract surgery since the inception of phacoemulsification, diagnosing and managing posterior capsular rent still remains a challenge. Identification of the pre-existing risk factors is of utmost importance so that precautionary modifications are done in the surgery to prevent this complication. Once it happens, timely realization and appropriate management can have excellent outcomes.
\end{abstract}

\section{Keywords}

Posterior Capsular Rent, Vitreous Loss, Traumatic Cataract, Small Pupil, Pseudoexfoliation, Vitrectomy, Triamcinolone Acetonide

\section{Introduction}

Posterior capsule rent (PCR), reported to occur in $0.5 \%$ to $7.5 \%$ of cases is a significant potential complication of cataract surgery. An improperly managed PCR can have an adverse impact on the outcome of cataract surgery. Vitreous loss appears to be the most crucial factor determining the eventual visual outcome.

PCR, with or without vitreous disturbance leads to increased incidence ofhyphema, increased persistent post operative inflammation, retained cortex, corneal edema, post operativeendophthalmitis, cystoid macular edema (if chronic leading to macular pseudoholes) and tractional retinal detachment in long standing cases.

\section{Risk Factors}

Certain predisposing factors are posterior polar cataract [1]-[4] (with a pre-existing posterior capsular dehis-

*Corresponding author.

How to cite this paper: Rani, N. and Gupta, P. (2014) Posterior Capsular Rent: Risk Factors, Diagnosis and Management. Surgical Science, 5, 224-226. http://dx.doi.org/10.4236/ss.2014.55038 
cence), traumatic cataract, mature cataract, hypermature cataract, post-vitrectomy cataract, eyes with long axial length (with weak bag and weak zonules), eyes with short axial length (with crowded anterior chamber), pseudoexfoliation cases (with weak bag, weak zonules and poorly dilating pupil) [1]-[3].

Intraoperative factors may be poor visibility due to deep set eyes with prominent brow, fluid pooling, dense arcus, corneal scars, small pupil (as in diabetic patients, post-uveitic posterior synechiae, pseudoexfoliation, traumatic cataracts and senile pupillary rigidity). Small pupil must be dealt with intacameral adrenaline, multiple sphincterotomies, stretch pupilloplasty or iris hooks [1] [2]. Intraoperative events resulting in posterior capsular rent-

*Extension of radial tears of the anterior continuous curvilinear capsulorhexis through the capsular fornix into the posterior capsule.

*Vigorous hydrodissection specially in incomplete rhexis in cases like posterior polar cataract, traumaticcataract, pseudoexfoliationcases, mature and hypermature cataract.

*Occurs during removal of last fragment of nucleus following a transient post-occlusion surge)in phacoemulsification.

*Infrequently during intraocular lens placement and dialling.

*In case of incomplete capsulorrhexis, during irrigation aspiration of cortex tags of anterior capsule can be caught into the probe.

*Hydroprolapse of the nucleus or hooking out of the nucleus through a small rhexis can exert undue pressure on the posterior capsule (in small incision cataract surgery).

*Manipulation in the bag without adequately pressurizing the anterior chamber. When the anterior chamber keeps collapsing there are high chances of the lax posterior capsule coming up and getting caught.

*During polishing of the posterior capsule.

*Direct hit by phacoemulsification probe, chopper or dialer.

\section{Diagnosis}

Early recognition of a posterior capsule rent is pertinent to its successful management eventually resulting in good visual outcome.

Signs of a posterior capsule rent which should alert a surgeon are [1]:

*Sudden deepening of the anterior chamber.

*Momentary dilatation of the pupil (snap sign).

* Sudden brightening of the fundal glow.

*Nucleus paradoxically moves away on aspiration,sways sideways or falters posteriorly away from the nucleus.

*Tiiting of one pole of the nucleus.

*Nuclear fragments which were previously mobile, lose their mobility.

*Whole of the nucleus or a part of it may suddenly sink or disappear posteriorly into the vitreous cavity.

\section{Management}

Once the surgeon realizes a posterior capsular rent, thephaco probe shouldnot be withdrawn instantly in a haste. First inject the viscoelastic with the non dominant hand through the sideport to inflate the bag properly and then withdraw. This small precaution will prevent the anterior chamber from collapsing eventually leading to enlargement of the rent, disruption of the anterior hyaloid face and prolapse of the vitreous into the anterior chamber. So maintenance of the anterior chamber throughout the management of the posterior capsular rent is of prime importance to prevent further complications.

Next step is to confirm the presence of vitreous prolapsed into the anterior chamber. It can be assessed by following simple maneuvers-

*Sweep a sponge or swab stick along the site of incision. Vitreous if present can be seen as strands along the section.

*Sweep the spatula from the anterior chamber angle under the incision towards the rent. Vitreous if present will be seen getting dragged as it has a tendency to come towards the wound.

*Stain with triamcinolneacetonide just adjacent to the margin of the rent. Vitreous if present will get stained.

Next step is to decide about the management of the nuleus. It will depend on the extent of the rent and size, 
position and hardness of nucleus.

If the rent is small and a soft nucleus is left, with the help of a second instrument it can be moved away from the site of rent and phacoemulsification can be continued. Aspiration flow rate should be low. Vacuum should also be low to prevent post-occlusion surge. Use of lens glide has been advocated by some to facililtate this step.

If a large brunescent nucleus is left its better to convert to a large incision sugery.

Residual cortex and epinucleus should be removed using low flow, low vacuum bimamual irrigation aspiration technique. cortex remote from the rent should be stripped towards the rent site and not vice-versa. An alternative method is "dry aspiration" [5] [6]. Epinuclear sheet can also be taken out through viscoexpression.

If the nucleus has descended partially into vitreous, its prudent not to go chasing behind it. Alternatively, posterior assisted levitation technique can be used to bring the nucleus into the papillary plane and anterior chamber through the pars plana route for further management. Retrieval of nucleus from deep vitreous isnot recommended and should be dealt with after a suitable interval by a vitreoretinal surgeon.

Anterior vitrectomy must be carried out meticulously to prevent a myriad of post operative complications like vitreous wick syndrome,vitreous touch syndrome, endophthalmitis, cystoids macular edema, glaucoma and tractional retinal detachment.

Sponge vitrectomy is now obsolete because of the intense vitroretinal traction it used to exert.

Automated vitrectomy should be done [5] [6]). Vitrectomy probe should never be inserted through the main wound. Instead a separate $1 \mathrm{~mm}$ incision should be made. It should be started at the section and then proceed inwards. Maximum possible cutting rates (upto800 or more) with low vacuum and flow rate should be used.

Bimanual technique for infusion during vitrectomy is a better option than coaxial cannulas. Coaxial infusion canulas have a tendency to enlarge the rent as well as it hydrates the vitreous to a greater extent.

Vitrectomy tip should go at least 1-2 mm behind the posterior capsule. The aspiration port is directed upwards towards the cornea.

Alternatively, few advocate 23gauge or 25 gauge pars planavitrectomy as it doesnot pull the vitreous anteriorly and transects the vitreous at its base which lessens the chance of post-operative vitreoretinaltractional components to a greater extent.

For confirming the completion of vitrectomy, following simple maneuvers can be used-

*Inject air bubble and look for its fragmentation.

*Inject pilocarpine and look for peaking of pupil.

*Inject triamcinolneacetonide which will stain the vitreous.

In cases where posterior capsule rent has occurred but anterior vitreous phase is intact, a prophylactic peripheral iridectomy must be done.

Next step is the placement of intraocular lens. It again depends on the site and extent of rent. It is better to place a 3-piece lens in sulcus. If the rent is small lens can also be placed into the bag without much dialing. Whether in bag or in sulcus the haptics should be oriented 90 degrees away from the rent. After placing the lens check for its stability and centration. If required, we have to opt for iris-fixation or scleral fixation of the lens.

\section{References}

[1] Osher, R.H. and Cionni, R.J. (1990) The Torn Posterior Capsule: Its Intraoperative Behaviour, Surgical Management and Long Term Consequences. Journal of Cataract \& Refractive Surgery, 16, 490-494. http://dx.doi.org/10.1016/S0886-3350(13)80805-X

[2] Moreno, J., Duch, S. and Lajara, J. (1993) Pseudoexfoliation Syndrome: Clinical Factors Related to Capsular Rupture in Cataract Surgery. Acta Ophthalmologica, 71,181-184.

[3] Guzek, J.P., Holm, M., Cotter, J.B., Cameron, J.A., Rademaker, W.J. and Wissinger, D.H. (1987) Rick Factors for Intraoperative Complications in 1000 Extracapsular Cases. Ophthalmology, 94, 461-466. http://dx.doi.org/10.1016/S0161-6420(87)33424-4

[4] Osher, R.H., Yu, B.C. and Koch, D.D. (1990) Posterior Polar Cataracts: A Predisposition to Intraoperative Posterior Capsular Rupture. Journal of Cataract \& Refractive Surgery, 16, 157-162. http://dx.doi.org/10.1016/S0886-3350(13)80724-9

[5] Basti, S., Vasavada, A.R., Thomas, R. and Padmanabhan, P. (1993) Extracapsular Cataract Extraction: Surgical Techniques. Indian Journal of Ophthalmology, 41, 19-21.

[6] Thomas, R., Kuriakose, T. and George, R. (2000) Towards Achieving Small Incision Cataract Surgery 99.8\% of the Time. Indian Journal of Ophthalmology, 48, 145-151 [PubMed]. 